\title{
Orbital degree of freedom in artificial electron lattices on metal surface
}

\author{
Liang Ma目 Wen-Xuan Qiu图 Jing-Tao Lü̈ and Jin-Hua Gad \\ School of Physics and Wuhan National High Magnetic Field Center, \\ Huazhong University of Science and Technology, Wuhan 430074, P. R. China
}

\begin{abstract}
Orbital degree of freedom plays a fundamental role in condensed matter physics. Recently, a new kind of artificial electron lattice has been realized in experiments by confining the metal surface electrons with adsorbed molecular lattice. A most recent example is the Lieb lattice realized by CO adsorption on $\mathrm{Cu}(111)$ surface [M. R. Slot, et al., Nat. Phys. 13, 672 (2017)]. The Lieb lattice is of special interest due to its flat band physics. Here, by first-principles calculations, muffin-tin potential model and tight binding model, we demonstrate that, the high energy states observed in the experiment actually correspond to the artificial $p$-orbitals of the electron lattice. Our numerical results, together with the experimental observation, show that artificial $p$-orbital fermionic lattice has already been realized in solid state system. This opens a new avenue to investigate the orbital degree of freedom in a controllable way.
\end{abstract}

The orbital degree of freedom, which refers to the orbital degeneracy and orientational anisotropy, is a fundamental attribute of the Bloch electrons in crystal, in addition to their charge and spin. When coupled to the charge and spin, orbital degree of freedom of electrons can give rise to many important phenomena, such as metal-insulator transition, superconductivity, and colossal magneto-resistance [1, 2]. However, understanding the orbital physics in real materials is still a big challenge due to some realistic reasons, e.g, the constraints of materials, the coupling among multiple degrees of freedom and the lack of controllability.

Utilizing artificial quantum systems is a promising way to study the orbital physics. The most successful example is the cold atoms in optical lattices, where atoms can be excited into higher orbital states of the optical lattice. In last decade, $p$-orbital related novel quantum states in optical lattices have been intensively studied (see, for example, Ref. 3 and the references therein). For example, for $p$-band bosons, an unconventional BEC has been realized in experiment [4 6]. However, an experimental realization of $p$-band fermions has not been reported in any artificial quantum lattices so far.

Recently, a new kind of artificial two dimensional electron lattice has been realized in experiments. The first example is the molecular graphene 7, [8], where the $\mathrm{Cu}$ surface electrons, a perfect two dimensional electron gas (2DEG) with $k^{2}$ dispersion, is confined in a honeycomb lattice by lateral periodic potential induced by adsorbed molecules. Even more exciting is the realization of artificial Lieb lattice 9 11, that has not been found in natural materials and is of special interest due to its flat band physics. These pioneering works demonstrate that artificial electron lattice on metal surface could be a promising solid-state quantum simulation platform.

An interesting issue in the recent experiment [10] is that some high-energy electron states with complex local density of states (LDOS) pattern are observed in the scanning tunnelling microscope (STM), which are drastically different from the low energy states. Here, combining first-principles simulation based on Density Functional Theory (DFT), muffin-tin potential model and tightbinding calculations, we demonstrate that these high energy states originate from artificial $p$-orbitals of the electron lattice. Through carefully comparison of our theoretical calculation with the experimental observation, we show that the experiment in Ref. 10 has actually realized an artificial $p$-orbital square (and Lieb) electron lattice in solid state system. It may give the first example of the two dimensional $p$-orbital fermion lattice in artificial quantum systems, and offers an ideal solid state platform to study orbital physics in a controllable way.

Let us start with the square electron lattice on metal surface. The structure is shown in Fig. 1 (a), where the $\mathrm{CO}$ molecules (black balls) are arranged on the $\mathrm{Cu}(111)$ surface to form a square lattice. Note that, due to the geometry of the $\mathrm{Cu}(111)$ surface, the lattice constant (CO molecule lattice) in $x$ direction $a_{0}$ is slightly different from that in $y$ direction $b_{0}$. For simplicity, we ignore this difference in the muffin-tin potential model unless specified otherwise. Here, each CO molecule exerts a repulsive potential on the $\mathrm{Cu}$ surface electrons, which can be described by a muffin-tin potential $U(r)$ [see Fig. 1 (a), $U_{0}>0$ inside the blue circles and zero elsewhere, $d$ is the diameter of circular potential]. The Hamiltonian of the $\mathrm{Cu}$ surface states now is

$$
H_{\mathrm{Cu}}=\frac{\hbar^{2}}{2 m^{*}} \boldsymbol{k}^{2}+U(r)
$$

where $m^{*}=0.38 m_{e}$ is the effective mass of the $\mathrm{Cu}$ surface electrons, and $U_{0}=9 \mathrm{eV}, d=0.5 \mathrm{~nm}$ are reasonable values for the muffin-tin potential of $\mathrm{CO} / \mathrm{Cu}(111)$ system [12, 13]. It is actually an anti-dot lattice, since the surface electrons under the $\mathrm{CO}$ molecules are depleted by the repulsive potential $U(r)$. Consequently, surface electrons are forced into a square lattice, where the electron sites are in the center of the squares formed by four adjacent $\mathrm{CO}$ molecules. Theoretically, the corresponding band structure can be obtained by solving the Hamiltonian of the muffin-tin potential model with plane wave 


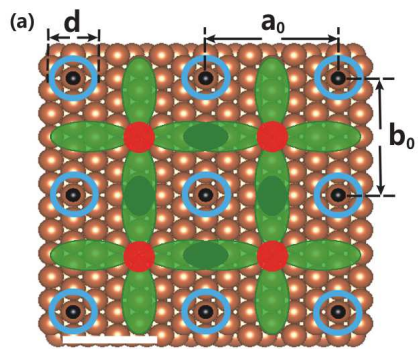

(e)

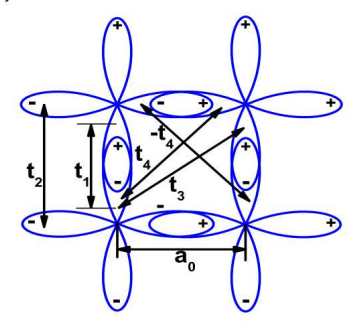

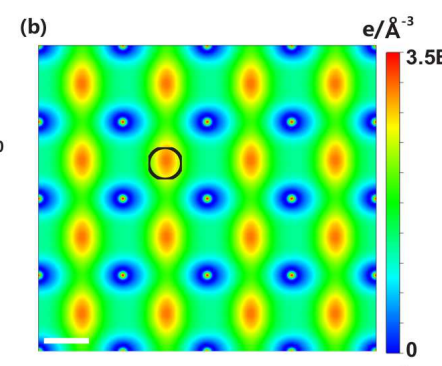

(i)

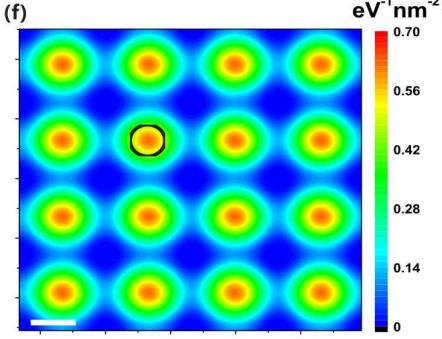

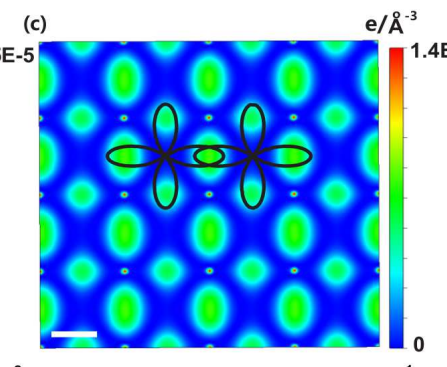

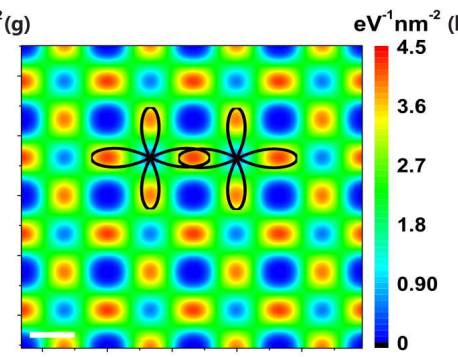

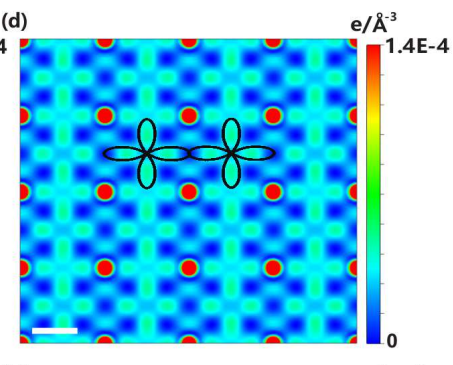

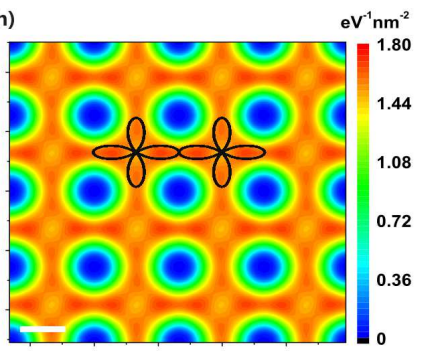

FIG. 1. (Color online) (a) Schematic of the artificial electron square lattice on $\mathrm{Cu}(111)$ surface. The red discs are the $s$-orbitals and the green lobes denote the $p$-orbitals. The lattice vectors are $a_{0} \approx 1.33 \mathrm{~nm}$ and $b_{0} \approx 1.28 \mathrm{~nm}$, respectively. (b),(c),(d) are the DFT simulated STM images, where the energy region, with respect to the Fermi level, are $-0.3 \sim-0.2 \mathrm{eV},-0.1 \sim-0.08$ $\mathrm{eV}$ and $0.55 \sim 0.65 \mathrm{eV}$, respectively. (e) Schematic of the $p$-orbital tight binding model on square lattice. (f),(g),(h) are the LDOS calculated from the muffin-tin potential model, where the energy are $1.14 \mathrm{eV}, 1.47 \mathrm{eV}$ and $2.15 \mathrm{eV}$, respectively. $a_{0}=1.33 \mathrm{~nm}, b_{0}=1.28 \mathrm{~nm}, U_{0}=9 \mathrm{eV}, d=0.5 \mathrm{~nm}$. Scale bars, $1 \mathrm{~nm}$.

basis. Here, the LDOS of surface electrons is the most important quantity, which actually represents the electron wave functions and can be directly measured by STM. Theoretically, the LDOS can be calculated from

$$
\operatorname{LDOS}(r, \varepsilon)=\sum_{n k \sigma}\left|\phi_{n k \sigma}(r)\right|^{2} \delta\left(\varepsilon-\epsilon_{n k \sigma}\right),
$$

where $\phi_{n k \sigma}(r)$ is the wave function of surface electron, $n$, $k$ and $\sigma$ are the indices of band, momentum, and spin, respectively.

In addition to the muffin-tin potential model, we perform DFT calculations to direct simulate the experimentally realized system[13]. We use the Vienna Ab-initio Simulation Package (VASP), which is based on the projector-augmented wave method and a plane wave basis set 14]. We choose the Perdew-BurkeErnzerhof (PBE) version of the generalized gradient approximation [15], and the energy cutoff is $400 \mathrm{eV}$. A four-layer slab is used to model the $\mathrm{Cu}(111)$ surface and a $10 \AA$ vacuum region separates slabs between nearest supercells to avoid their interaction. The positions of the $\mathrm{CO}$ molecules and the top layer $\mathrm{Cu}$ atoms are optimized in a smaller supercell and used in present calculation. We can directly simulate the STM image (LDOS of surface electron states) using the Tersoff-Hamann scheme [16] from the DFT electronic structure.

First, we show that the LDOS of this artificial square lattice observed in experiment can be well reproduced by the both muffin-tin potential model and the firstprinciples calculation. The numerical results are summa- rized in Fig 1, where Fig. 1(b),(c),(d) are results of firstprinciples calculation, and Fig. [ (f), (g), (h) are those from the muffin-tin potential model. Basically, three typical surface electron LDOS patterns at different energies have been observed in experiment: (1) at low energy [Fig. [1(b),(f)], the electron states are localized around the lattice sites, so we get a square lattice pattern; (2) for a high energy state [Fig. 1 (c),(g)], the electron states are now located in between the lattice sites; (3) increasing the energy further, though the electron states are still mainly in between the lattice sites, some fine structures of the LDOS pattern appear, i.e., we find a LDOS node between two adjacent lattice sites [Fig. 1(d),(h)]. Both DFT and the muffin-tin potential model nicely reproduce the experimental observations [Fig. 4 (e), (f), (g) in Ref. 10].

The main focus of this work is to understand the physical origin of these complex electron LDOS (wave functions) pattern. Our viewpoint here is that, the low energy states are from the $s$-band of the electron square lattice, while the high energy states are from the $p$-bands. In other words, even for this anti-dot lattice, the orbital degree of freedom is still a valid concept. Here, each electron lattice site could be considered as an artificial atom in two dimensions with various atomic orbitals, such as $s$-and $p$-orbitals (only $p_{x}$ and $p_{y}$ here since it is a two dimensional system). These artificial orbitals form the corresponding energy bands due to hopping between them. Thus, Fig. 1 (b-d) and (f-h) actually show the real space distribution of these orbital bands. Importantly, in Fig. 1 (c), we see that the $p$-orbitals of two neighboring sites 

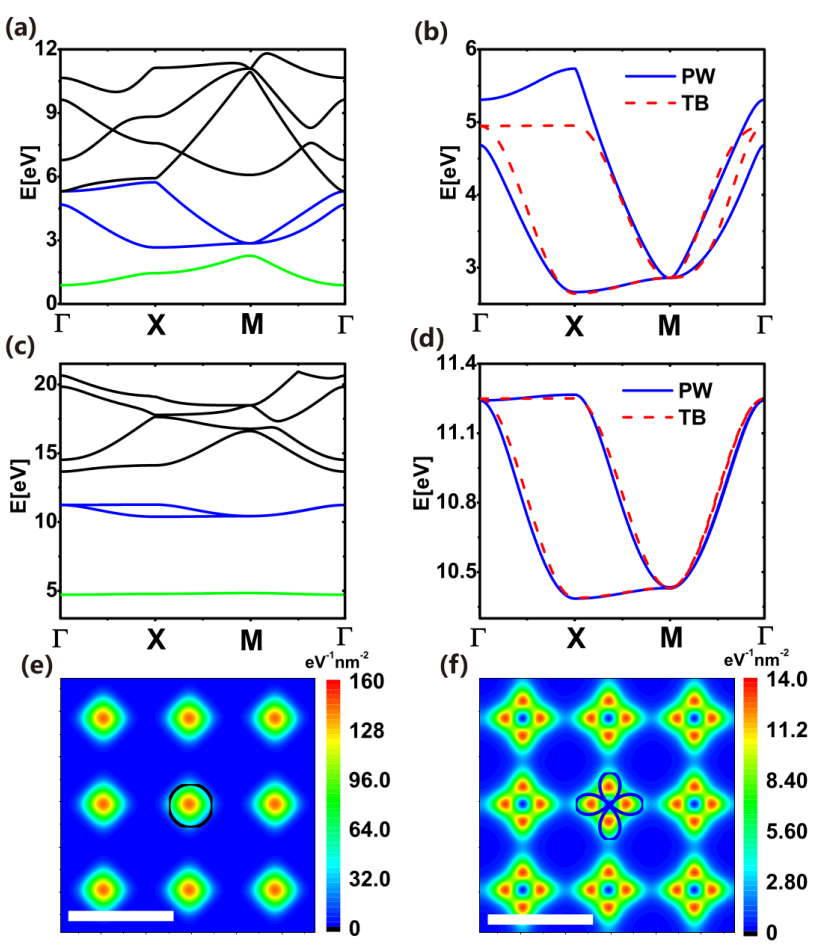

FIG. 2. (Color online) (a) Energy bands of the electron square lattice, where green lines are the $s$-bands, blue lines are the $p$-bands. $U_{0}=9 \mathrm{eV}, d=0.5 \mathrm{~nm}, a_{0}=0.95 \mathrm{~nm}$. (b) Fitting the $p$-bands in (a) (blue, solid) with a $p$-orbital tight binding model on square lattice (red, dashed). PW means the muffintin model with plane wave basis. $\left[t_{1}, t_{2}, t_{3}, t_{4}\right]=[0.55,-0.0275$, 0.0131, 0.0963] eV. $\varepsilon_{p_{x}}=\varepsilon_{p_{y}}=3.85 \mathrm{eV}$. (c) Energy bands of the electron square lattice in the atomic limit. $a_{0}=0.95$ $\mathrm{nm}, U_{0}=15 \mathrm{eV}$ and $d=0.9 \mathrm{~nm}$. (d) Fitting the $p$-bands in (c) (blue, solid) with the tight binding model (red, dashed). $\left[t_{1}, t_{2}, t_{3}, t_{4}\right]=[0.21,-0.0055,0.0026,0.0014] \mathrm{eV}, \varepsilon_{p_{x}}=\varepsilon_{p_{y}}=$ $10.83 \mathrm{eV}$. (e) LDOS of the $s$-bands in atomic limit, $E=4.8$ eV. (f) LDOS of the $p$-bands in atomic limit at $E=10.8 \mathrm{eV}$. Scale bars, $1 \mathrm{~nm}$.

form a $\sigma$ bond, which is localized in between the sites. Thus the STM measurements actually have observed the artificial $p$-orbital $\sigma$ bonds. Meanwhile, at higher energy, the $p$-orbitals form anti-bonding $\sigma^{*}$ bonds, which have function nodes in between the artificial atoms. This is the origin of the LDOS nodes observed in the STM measurement.

Given the success of our muffin-tin potential model, we further use it to calculate the energy bands of the square lattice in order to prove the above physical picture. We show that the calculated band structure can be well understood from the orbital point of view. The calculated bands are shown in Fig. 2 (a), where we set $a_{0}=b_{0}=0.95 \mathrm{~nm}$ and $U_{0}=9 \mathrm{eV}, d=0.5 \mathrm{~nm}$ are the parameters for $\mathrm{CO} / \mathrm{Cu}(111)$ system. Note that $a_{0}$ is the distance between molecules which is tunable in experiment. Different $a_{0}$ gives different hopping amplitude, but the corresponding band shape is similar. We see that there is one band with the lowest energy (green solid line) gapped from the others. This is the $s$-band, because in square lattice each site contributes one $s$-orbital and these $s$-orbitals form one $s$-band with lowest energy. The $s$-orbitals are around the lattice sites, and thus should give an electron square lattice. In our calculation, in the energy interval of the $s$-bands, the corresponding LDOS are all like Fig. 1 (b), which show a clear square lattice LDOS pattern and are consistent with the experimental observation. Note that the $s$-band from the muffin-tin potential calculation can be well described by a single band tight-binding model on square lattice. Similarly, the upper two bands above the $s$-band (blue solid lines) should be the $p$-bands, because each 2D atom has two $p$ orbitals, i.e. $p_{x}$ and $p_{y}$. We use a two-band $\left(p_{x}, p_{y}\right)$ tight binding model on square lattice, which is illustrated in Fig. 1 (e), to fit the $p$-bands. The tight binding Hamiltonian is

$$
H(k)=\left(\begin{array}{cc}
H_{p_{x}} & V_{p_{x} p_{y}} \\
V_{p_{y} p_{x}} & H_{p_{y}}
\end{array}\right)
$$

where

$$
\begin{aligned}
H_{p_{x}}= & \epsilon_{p_{x}}+2 t_{1} \cos \left(k_{x} a_{0}\right)+2 t_{2} \cos \left(k_{y} a_{0}\right) \\
& +4 t_{3} \cos \left(k_{x} a_{0}\right) \cos \left(k_{y} a_{0}\right), \\
H_{p_{y}}= & \epsilon_{p_{y}}+2 t_{1} \cos \left(k_{y} a_{0}\right)+2 t_{2} \cos \left(k_{x} a_{0}\right) \\
& +4 t_{3} \cos \left(k_{x} a_{0}\right) \cos \left(k_{y} a_{0}\right), \\
V_{p_{x} p_{y}}= & -4 t_{4} \sin \left(k_{x} a_{0}\right) \sin \left(k_{y} a_{0}\right) .
\end{aligned}
$$

Here, $V_{p_{x} p_{y}}=V_{p_{y} p_{x}}$. The nearest neighbour hopping $t_{1}$, $t_{2}$ and the next nearest neighbour hopping $t_{3}, t_{4}$ are considered, which are illustrated in Fig. 10(e). The fitting is quite well as shown in Fig 2 (b), except that the tight binding model can not reproduce the gap at the $\Gamma$ point. We attribute this difference to the band overlap with the $d$-bands. As can be seen in Fig. 2 (a), there is no gap between the $p$ - and $d$-bands. This means that there should be some overlap between them, which is not included in the tight binding model.

In order to make the $p$-orbital picture more clear, we further consider an extreme case in the muffin-tin potential model, where we set $a_{0}=0.95 \mathrm{~nm}$, and use a fictional muffin-tin potential $U_{0}=15 \mathrm{eV}$ and $d=0.9 \mathrm{~nm}$. With given $a_{0}$ and very large values of $U_{0}$ and $d\left(a_{0}>d\right)$, the hopping between adjacent sites is greatly suppressed, so that the artificial atoms are nearly isolated. Thus, it is just the atomic limit. The band structure in this atomic limit is given in Fig. 2(c). It is now obvious that the two $p$-bands are separated from the higher $d$-bands, i.e. there is no band overlap any more. As a consequence, the gap of the $p$-bands at the $\Gamma$ point disappears. The fitting of $p$-bands is given in Fig. 2 (d). The tight binding model now can give a perfect description of the $p$-bands.

We plot the LDOS pattern in the atomic limit in Fig. 2 (e) and (f). At low energy [Fig. 2(e)], we see that the 

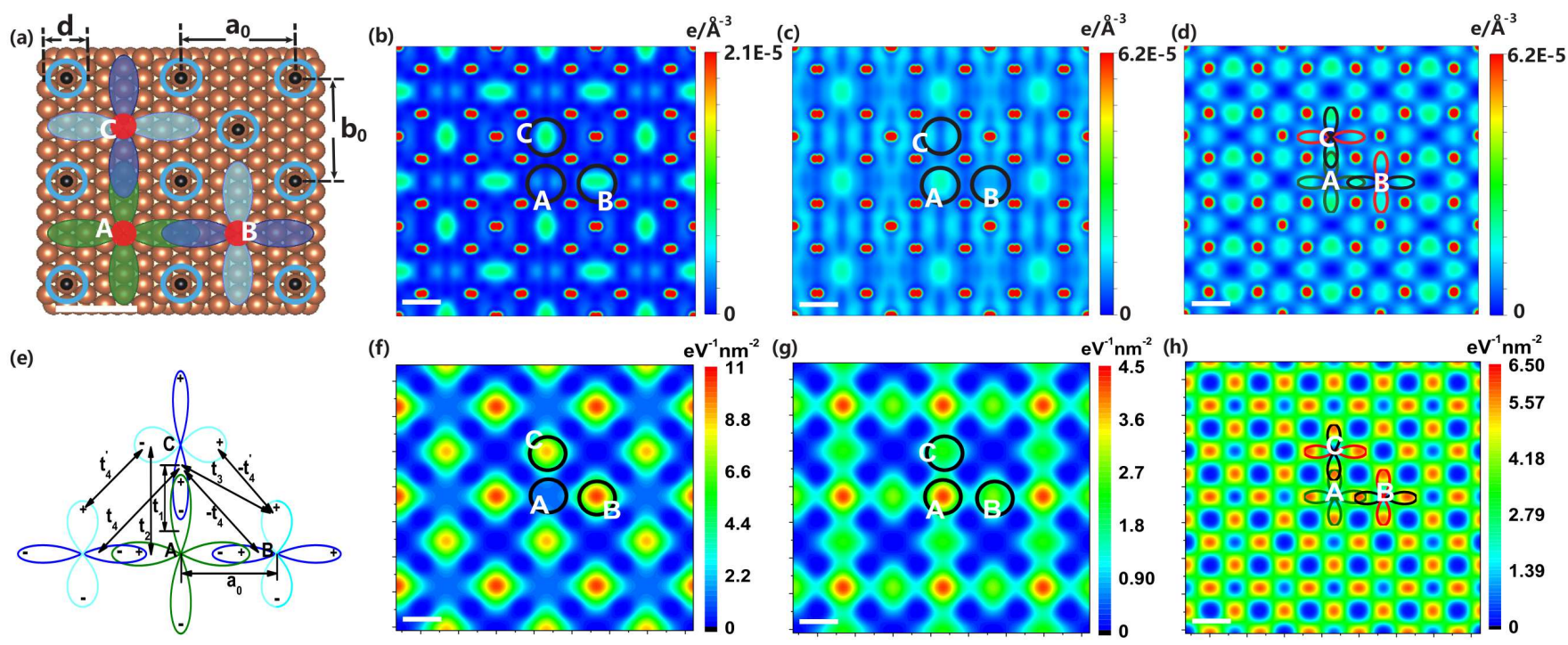

FIG. 3. (Color online) (a) Schematic of Lieb lattice. (b),(c),(d) are the DFT simulated STM image (LDOS), where the energy regions are $-0.5 \sim-0.4 \mathrm{eV},-0.2 \sim-0.1 \mathrm{eV}$ and $0.2 \sim 0.3 \mathrm{eV}$, respectively. $a_{0}=1.33 \mathrm{~nm}, b_{0}=1.28 \mathrm{~nm}$. (e) Schematic of the $p$-orbital tight binding model on Lieb lattice. The $p$-orbitals with different energy are denoted with different colors. (f),(g),(h) are the calculated LDOS with muffin-tin potential model, the energy of which are $0.61 \mathrm{eV}, 0.75 \mathrm{eV}$, and $1.29 \mathrm{eV}$, respectively. $a_{0}=1.33 \mathrm{~nm}, b_{0}=1.28 \mathrm{~nm}, U_{0}=9 \mathrm{eV}, d=0.5 \mathrm{~nm}$. Scale bars, $1 \mathrm{~nm}$.

isotropic s-orbitals are well separated from each other. Increasing the energy to the $p$-band region, we get an LDOS pattern in Fig. 2 (f), resembling the shape of isolated $p$-orbitals. All the discussions above give a clear illustration of the $p$-orbital picture in the square lattice.

Another important issue is the position of Fermi level relative to the $p$-bands, which depends on the square lattice constant $a_{0}$ [7, 9, 12. We can give an estimation about the value of $a_{0}$ to access the $p$-bands in square lattice. In square lattice, the number of electrons in each site is about $N_{e} a_{0}^{2}$, where $N_{e}$ is the electron density of the metal surface. For $\mathrm{Cu}(111)$ surface, $N_{e}$ is about 0.72 $\mathrm{nm}^{-2}$. Here, we assume that the adsorbed $\mathrm{CO}$ molecules do not modify the surface electron number of $\mathrm{Cu}$. It is reasonable for the experimental situation, since we do not expect that one hundred $\mathrm{CO}$ molecules can change the Fermi level of the bulk $\mathrm{Cu}$ crystal. To access the $p$-bands, $a_{0}$ should be in the region $1.7 \mathrm{~nm}<a_{0}<2.9 \mathrm{~nm}$. This estimation is consistent with the experimental observations. In experiment [10], two square lattices are realized. The $a_{0}$ of large one is about $2.56 \mathrm{~nm}\left(b_{0} \approx 2.66 \mathrm{~nm}\right)$, two times larger than that of the small one. So, according to the estimation above, the Fermi level of the large square lattice should be at the $p$-bands, while that of the small lattice is at the $s$-bands. In the STM measurement, for the large square lattice, the LDOS patterns of the $s$-band and bonding $p$-band are found below the Fermi level, while that of the anti-bonding $p$-band is above the Fermi level. This is consistent with our estimation. In contrast, for the small square lattice, no matter with positive or negative bias voltage, only the LDOS pattern of the $s$-band is observed, indicating that the Fermi level is at the $s$-band. In order to access the $p$-band, more larger bias voltage is needed for the small square lattice case.

Now, we turn to the Lieb lattice. As shown in Fig. 3 (a), there are three lattice sites (A, B,C) in each unit cell of a Lieb lattice. Thus, there are three $s$-bands with lowest energy, of which the middle one is a flat band if only the nearest neighbor hopping is considered. A unique property of this flat band is its special LDOS pattern. The electrons in the flat band are only localized at the $\mathrm{B}$ and $\mathrm{C}$ sites, while the electrons in other two $s$-bands mainly distribute around the A sites. This unique LDOS pattern actually reflects the flat band localization phenomenon in Lieb lattice, and has been observed in different systems [9, 10, 17, 18.

We first show that, for the $s$-bands, the special LDOS pattern of the flat band (the flat band localization phenomenon) can be well reproduced by the our calculation. The DFT results are shown in Fig. 3 (b) and (c), while the corresponding muffin-tin results are given in Fig. 3 (f) and (g) as a comparison. In Fig. B (b), it is seen that the electron states are absent around the A site, which is just the special LDOS pattern of the Lieb flat band. Increasing the energy to the upper $s$-band, the electrons should distribute mainly around the A site, instead of the $\mathrm{B}$ and $\mathrm{C}$ sites. This is observed in Fig. 3 (c). The muffintin potential model can also reproduce these features, as shown in Fig 3 (f), (g).

The $p$-orbital picture also works well for the high energy states in the Lieb lattice. It is not surprising because a square lattice can be changed into a Lieb lattice 
(a)

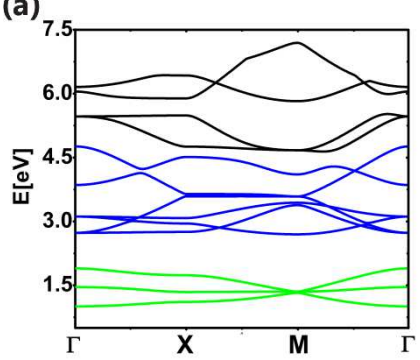

(b)

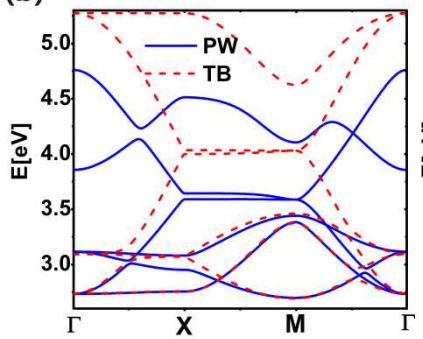

(c)

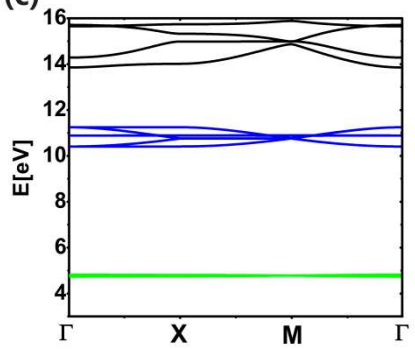

(d)

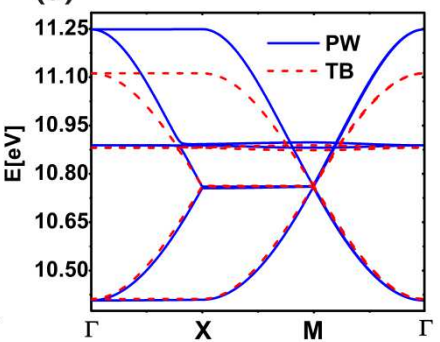

(e)

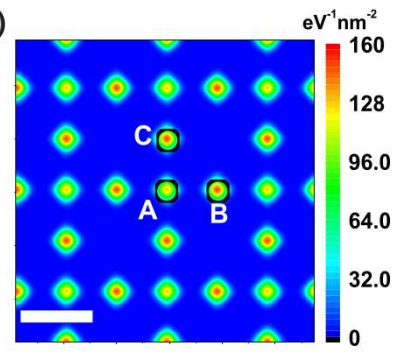

(f)

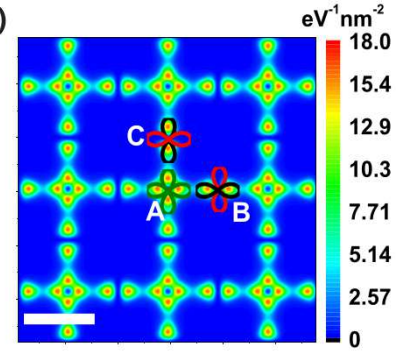

(g)

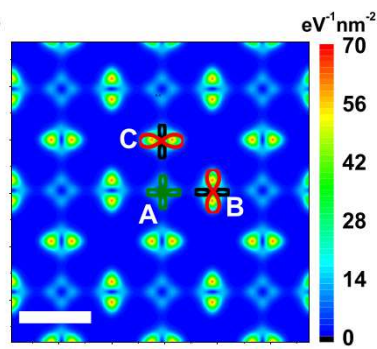

(h)

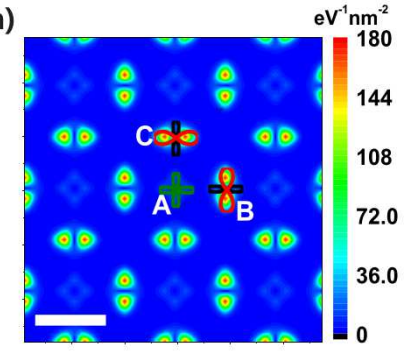

FIG. 4. (Color online) (a) Energy bands of the Lieb lattice. $a_{0}=0.95 \mathrm{~nm}, d=0.5 \mathrm{~nm}, U_{0}=9 \mathrm{eV}$. (b) Fitting the $p$-bands in (a) (blue, solid) with the $p$-orbital tight binding model (red, dashed) on Lieb lattice. $\left[t_{1}, t_{2}, t_{3}, t_{4}, t_{4}^{\prime}\right]=[0.63,-0.0345,0.011,0.1563,0.096]$ $\mathrm{eV} . \varepsilon_{\mathrm{A} p_{x}}=\varepsilon_{\mathrm{A} p_{y}}=4.03 \mathrm{eV}, \varepsilon_{\mathrm{B} p_{x}}=\varepsilon_{\mathrm{C} p_{y}}=4 \mathrm{eV}, \varepsilon_{\mathrm{B} p_{y}}=\varepsilon_{\mathrm{C}_{x}}=3.075 \mathrm{eV}$. (c) Energy bands of the Lieb lattice in the atomic limit. $a_{0}=0.95 \mathrm{~nm}, d=0.9 \mathrm{~nm}, U_{0}=15 \mathrm{eV}$. (d) Fitting the $p$-bands in (c) (blue, solid) with the $p$-orbital tight binding model (red, dashed) on Lieb lattice. $\left[t_{1}, t_{2}, t_{3}, t_{4}, t_{4}^{\prime}\right]=[0.175,-0.0055,0.0026,0.0016,0.0019] \mathrm{eV} . \varepsilon_{\mathrm{A} p_{x}}=\varepsilon_{\mathrm{A} p_{y}}=10.761 \mathrm{eV}$, $\varepsilon_{\mathrm{B} p_{x}}=\varepsilon_{\mathrm{C} p_{y}}=10.763 \mathrm{eV}, \varepsilon_{\mathrm{B} p_{y}}=\varepsilon_{\mathrm{C} p_{x}}=10.881 \mathrm{eV}$. (e)-(h) The LDOS of the Lieb lattice in the atomic limit. The energies are taken at $4.8 \mathrm{eV}, 10.6 \mathrm{eV}, 10.8 \mathrm{eV}, 10.9 \mathrm{eV}$, respectively. Scale bars, $1 \mathrm{~nm}$.

by removing one of the four sites. The illustration of the p-orbitals in Lieb lattice is given in Fig. 3 (e). Similar to the square lattice, we expect that the $p$-band electrons will mainly distribute in between the sites, due to the formed $p$-orbital $\sigma$ bond, as was found in the experiment. The calculated LDOS confirms our expectation. We can see that, the experimental observation, the first-principles calculation [Fig. 3(d)], and the muffin-tin model [Fig. 3 (h)] all coincide well with the $p$-orbital picture. The main distinction from the square lattice is that the $p$-orbitals at the $\mathrm{A}, \mathrm{B}, \mathrm{C}$ sites are now inequivalent, due to their different geometric environment. They thus have different on-site energies, which are denoted with different colors in Fig. 3 (e).

We plot the band structure of the Lieb lattice calculated from the muffin-tin potential model with plane wave basis in Fig. 团 We first consider a normal situation [Fig. 4 (a)], where $a_{0}=0.95 \mathrm{~nm}, U_{0}=9 \mathrm{eV}$. In this case, we see that the lowest three bands (green solid lines) are $s$-bands of Lieb lattice. Considering the $p$-orbitals, there should be six $p$-bands above the three $s$-bands. This is shown in Fig. 4 (a) as blue solid lines, separated from both the lower $s$-bands and the upper $d$-bands. These $p$-bands can be qualitatively interpreted by a $p$-orbital tight binding model on Lieb lattice [see in Fig. 3 (e)]. In Fig. 4 (b), we show the tight binding fitting of the band structure. At low energy, the tight binding model works quite well, but it can not describe the top most two $p$-bands. Similar to the square lattice, we attribute this discrepancy to the influence of the upper $d$-bands. The corresponding LDOS of the $p$-bands are like Fig. 3 (d),(h), where the $p$-orbital $\sigma$ bonds are shown clearly.

We now consider the atomic limit of the Lieb lattice $\left(U_{0}=15 \mathrm{eV}, d=0.9 \mathrm{~nm}, a_{0}=0.95 \mathrm{~nm}\right)$ to support our $p$ orbital picture. In the atomic limit, the hopping between adjacent sites is greatly suppressed. The bands of the atomic limit are shown in in Fig. 4 (c). In this case, the $p$-bands are far from the $s$ - and $d$-bands. In Fig. 4 (d), we also use the $p$-orbital tight binding model to fit these $p$ bands. Now the agreement is much better. Since the next nearest neighbor hopping becomes very tiny here, some $p$-orbitals on the $\mathrm{B}$ and $\mathrm{C}$ sites now form dangling bonds, which results in degenerate flat $p$-bands. The LDOS in atomic limit are also given in Fig. 4. At low energy, the $s$-orbitals are around the lattice sites, and form a Lieb lattice [Fig. 田 (e)]. Note that, because the hopping is tiny in the atomic limit, the artificial atoms are nearly isolated. Thus, the unique LDOS pattern of flat band we mentioned above can not be observed here. Continuously increasing the energy, the LDOS can sequentially show different $p$-orbitals as illustrated in Fig. [4 (f), (g) and (h). As we mentioned above, this is because the energy of the $p$-orbitals in Lieb lattice are different.

In summary, we theoretically demonstrate that the high energy states in the artificial electron square (Lieb) lattice, as observed in the recent STM experiment, are 
from the $p$-bands of the artificial atom confined in the lattice. The orbital degree of freedom is still a valid concept in this artificial electron lattice system. Our results suggest that, the electron lattice realized in Ref. 10 may be the first artificial $p$-orbital fermionic system in the solid state. Compared with other artificial quantum systems, this kind of electron lattice on metal surface is easy to manipulate, and the electron states can be directly detected. Thus, we believe that it is an ideal solid state platform to study orbital physics. Finally, we comment that, the same physics applies to artificial antidot lattice on 2DEG in semiconductor heterostructures [19 21].

J.H.G. and J.T.L. are supported by the National Natural Science Foundation of China (Grants No. 11534001, $61371015,11274129)$.

* These two authors contributed equally

$\dagger$ jtlu@hust.edu.cn

¥ jinhua@hust.edu.cn

[1] Y. Tokura and N. Nagaosa, Science 288, 462 (2000).

[2] T. Hotta, Rep. Prog. Phys. 69, 2061 (2006)

[3] X. Li and W. V. Liu, Rep. Prog. Phys. 79, 116401 (2016)

[4] T. Müller, S. Fölling, A. Widera, and I. Bloch, Phys. Rev. Lett. 99, 200405 (2007).

[5] G. Wirth, M. Ölschläger, and A. Hemmerich, Nat. Phys. 7, 147 (2011).

[6] C. Wu, Mod. Phys. Lett. B 23, 1 (2009).

[7] K. K. Gomes, W. Mar, W. Ko, F. Guinea, and H. C.
Manoharan, Nature 483, 306 (2012).

[8] S. Wang, L. Z. Tan, W. Wang, S. G. Louie, and N. Lin, Phys. Rev. Lett. 113, 196803 (2014)

[9] W.-X. Qiu, S. Li, J.-H. Gao, Y. Zhou, and F.-C. Zhang, Phys. Rev. B 94, 241409 (2016)

[10] M. R. Slot, T. S. Gardenier, P. H. Jacobse, G. C. P. van Miert, S. N. Kempkes, S. J. M. Zevenhuizen, C. M. Smith, D. Vanmaekelbergh, and I. Swart, Nat. Phys. 13, 672 (2017).

[11] R. Drost, T. Ojanen, A. Harju, and P. Liljeroth, Nat. Phys. 13, 668 (2017).

[12] S. Li, W.-X. Qiu, and J.-H. Gao, Nanoscale 8, 12747 (2016)

[13] M. Ropo, S. Paavilainen, J. Akola, and E. Räsänen, Phys. Rev. B 90, 241401 (2014).

[14] G. Kresse and J. Furthmüller, Phys. Rev. B 54, 11169 (1996)

[15] J. P. Perdew, K. Burke, and M. Ernzerhof, Phys. Rev. Lett. 77, 3865 (1996)

[16] J. Tersoff and D. R. Hamann, Phys. Rev. Lett. 50, 1998 (1983).

[17] R. A. Vicencio, C. Cantillano, L. Morales-Inostroza, B. Real, C. Mejía-Cortés, S. Weimann, A. Szameit, and M. I. Molina, Phys. Rev. Lett. 114, 245503 (2015)

[18] S. Mukherjee, A. Spracklen, D. Choudhury, N. Goldman, P. Öhberg, E. Andersson, and R. R. Thomson, Phys. Rev. Lett. 114, 245504 (2015)

[19] C.-H. Park and S. G. Louie, Nano Lett. 9, 1793 (2009).

[20] M. Polini, F. Guinea, M. Lewenstein, H. C. Manoharan, and V. Pellegrini, Nat. Nanotechnol. 8, 625 (2013).

[21] M. Gibertini, A. Singha, V. Pellegrini, M. Polini, G. Vignale, A. Pinczuk, L. N. Pfeiffer, and K. W. West, Phys. Rev. B 79, 241406 (2009). 